\title{
BMJ Open Androgen deprivation therapy and the risk of iron-deficiency anaemia among patients with prostate cancer: a population-based cohort study
}

\author{
Fang-Jen Wu, ${ }^{1}$ I-Hsun Li, ${ }^{2,3,4}$ Wu-Chien Chien, ${ }^{5,6,7}$ Jui-Hu Shih, ${ }^{2,3}$ Yi-Chun Lin, ${ }^{8}$ \\ Chin-Min Chuang, ${ }^{9}$ Yih-Dih Cheng, ${ }^{10}$ Li-Ting Kao (i) $2,3,5,7$
}

To cite: Wu F-J, Li I-H, Chien W-C, et al. Androgen deprivation therapy and the risk of iron-deficiency anaemia among patients with prostate cancer: a population-based cohort study. BMJ Open 2020;10:e034202. doi:10.1136/ bmjopen-2019-034202

- Prepublication history and additional material for this paper are available online. To view these files, please visit the journal online (http://dx.doi. org/10.1136/bmjopen-2019034202).

Y-DC and L-TK contributed equally.

Received 10 September 2019 Revised 18 February 2020 Accepted 05 March 2020
Check for updates

(C) Author(s) (or their employer(s)) 2020. Re-use permitted under CC BY-NC. No commercial re-use. See rights and permissions. Published by BMJ.

For numbered affiliations see end of article.

Correspondence to Professor Li-Ting Kao; kaoliting@gmail.com

\section{ABSTRACT}

Objectives The administration of androgen deprivation therapy (ADT) to patients with metastatic prostate cancer might be associated with some adverse effects such as anaemia; however, few studies have been performed in East Asian populations. This study aimed to investigate the association between ADT and iron-deficiency anaemia (IDA) among patients with prostate cancer in a populationbased nationwide cohort.

Design Cohort study.

Setting Taiwan.

Participants Data for the cohort study were retrieved from the Taiwan National Health Insurance Research Database. Propensity score matching was used to select 7262 patients with prostate cancer who received ADT as the study group and 3631 patients who did not receive ADT as the control group.

Primary and secondary outcome measures This study individually tracked patients over a 3-year study period and identified those who were subsequently diagnosed with IDA following the index date.

Results The incidence rates of IDA in the study and control groups were 1.66 (95\% $\mathrm{Cl} \mathrm{Cl} 1.45$ to 1.86$)$ and 1.01 per 100 person-years $(95 \% \mathrm{Cl} 0.78$ to 1.25$)$, respectively. Furthermore, proportional Cox regression revealed an $\mathrm{HR}$ of $1.62(95 \% \mathrm{Cl} 1.24$ to 2.12$)$ for IDA in the study group after adjusting for patients' age, monthly income, geographic location, residential urbanisation level and incidence of hyperlipidaemia, diabetes, hypertension, coronary heart disease, inflammatory bowel disease, other cancers and gastrointestinal bleeding.

Conclusion Compared with its non-use among patients with prostate cancer, ADT use was associated with a higher risk of IDA.

\section{INTRODUCTION}

Prostate cancer (PCa) is the leading cause of cancer in men, and it accounted for 293000 deaths globally in $2013 .{ }^{12}$ In 2018 , an estimated 164690 new diagnoses in men and 29430 deaths from the disease were reported in the USA. ${ }^{3}$ Androgen deprivation therapy (ADT), also known as hormone therapy, has been a fundamental component of metastatic
Strengths and limitations of this study

- Data for this cohort study were retrieved from a large Asian population-based dataset in Taiwan.

- This study used a propensity score matching strategy to eliminate potential biases such as patients' demographics and comorbidities between the study and control cohorts.

- The sample size and statistical power were sufficient in this study.

- Selection bias may have been eliminated by the use of a population-based dataset.

- Information on personal history and lifestyle was unavailable when data were retrieved.

PCa management for more than half a century. ${ }^{4}$ This therapy can improve long-term survival for many patients. ${ }^{56}$ However, the decreased serum levels of endogenous androgen associated with ADT might result in some adverse effects, including decreased muscle mass, increased insulin resistance and fall risk, among patients with $\mathrm{PCa} .^{7-12}$

Iron-deficiency anaemia (IDA) is a common disease that affects approximately $5 \%$ of women and $2 \%$ of men in the USA. $^{13}$ Increasing numbers of studies have recognised that anaemia may have a potentially detrimental impact on the quality of life and survival of patients with PCa. ${ }^{14-17} \mathrm{~A}$ previous study (InCHIANTI study) further suggested that androgen deficiency contributes to the development of anaemia in men with $\mathrm{PCa}^{18}$ Some studies proposed that androgens may stimulate erythropoiesis through direct effects on erythroid progenitor cells and indirect inhibitory effects on hepcidin. ${ }^{19-21}$ Therefore, it is plausible that ADT may be associated with the incidence of IDA.

Accordingly, the association between ADT and anaemia has been reported in 
several western studies using Caucasian populations. ${ }^{22-24}$ However, to date, few studies have been conducted in East Asian populations even though the hereditary risk of PCa substantially varies by ethnicity and geography. ${ }^{25}{ }^{26} \mathrm{In}$ addition, the haemoglobin concentration, which is a critical indicator of anaemia, is recognised to vary by race/ ethnicity, lifestyle, demographics and other variables. ${ }^{27}$ Furthermore, a recent study of 32 patients receiving ADT demonstrated that haematological toxicities such as anaemia were more frequent in Chinese patients with $\mathrm{PCa}$ than in their western counterparts. ${ }^{28}$ Consequently, this study examined whether ADT is associated with a subsequent risk of anaemia in patients with $\mathrm{PCa}$ by employing a propensity score matching strategy using an Asian population-based dataset in Taiwan.

\section{METHODS \\ Database}

Data for the retrospective cohort study were retrieved from the Taiwan National Health Insurance Research Database (NHIRD). The NHIRD, which derived from the Taiwan Bureau of National Health Insurance, consists of inpatient and ambulatory medical benefit claims from approximately 22 million enrollees, representing more than $99 \%$ of Taiwan's population. Therefore, this database provides scientists in Taiwan with an exclusive opportunity to analyse and track medical service use by enrollees since the beginning of the Taiwan National Health Insurance programme in 1995. This study was exempt from full revision by the Tri-Service General Hospital Institutional Review Board (TSGHIRB No 2-105-05-082) because the dataset used was blinded to the public for research purposes.

\section{Study sample}

This cohort study included study and control groups. For the study group, 28367 patients who received a first-time diagnosis of PCa (International Classification of Diseases Ninth Revision Clinical Modification (ICD-9-CM) code 185) between 1 January 2001 and 31 December 2010 were selected from the Registry of Catastrophic Illness Patient Database in NHIRD. In total, 454 patients younger than 40 years were excluded because the prevalence of PCa is extremely low in this age strata. The date of ADT administration (including gonadotropin-releasing hormone agonists, antiandrogens, ketoconazole and oestrogen; ATC codes L02AE03, L02AE02, L02AE04, L02BB03, G03HA01, L02BB01, J02AB02 and L02AA) was assigned as the index date for patients with PCa who subsequently received ADT treatment. Furthermore, the date of a randomly selected outpatient visit was assigned as the index date for the diagnosis of PCa for patients who did not receive ADT. Meanwhile, 1605 patients who had received a diagnosis of IDA (ICD-9-CM codes 280, 280.0, 280.1, 280.8 and 280.9) and 1533 patients who had undergone orchiectomy prior to their index date were excluded. As a result, 24775 patients with PCa remained in the study, including 20272 and 4503 patients with ADT use and non-use, respectively, during the 3-year study period. Specifically, 44.06, 26.06, 14.65 and $15.23 \%$ of the ADT prescriptions were for cyproterone, bicalutamide, flutamide and other types of medications, respectively. The average time from PCa diagnosis date to patient entry date for both non-users and users were 758.4 days and 796.9 days, respectively.

Propensity score matching was used to identify 7262 patients with PCa who received ADT. This methodology could eliminate the differences attributable to differences in patient demographics and medical history between the groups. We used propensity score matching using the Mahalanobis metric (caliper of $0.25 \mathrm{SD}$ of the propensity score). The matching variables included year of the entry date, age, monthly income, geographic location, residential urbanisation level (divided into five levels, with one being the most urbanised and five being the least urbanised), ${ }^{29}$ hyperlipidaemia, diabetes, hypertension, coronary heart disease and inflammatory bowel disease. We performed a 2:1 matching study, because increasing sample size of cases or controls could increase the statistic power of the findings. Ultimately, 10893 patients were enrolled in this study, including 7262 who received ADT and 3631 who did not receive ADT. We categorised the patients receiving ADT into two levels according to the median duration of ADT use (median=144 days). Those patients who received ADT $<144$ days were identified as short-term ADT users. Moreover, patients receiving ADT $\geq 144$ days were defined as long-term ADT users. The power for this study was adequate (power $>0.9$ ). Each patient was subsequently followed individually for a 3-year period to identify those who received an IDA diagnosis following the index date.

\section{Statistical analysis}

All statistical analyses were performed using the SAS System for Windows V.9.4 (SAS Institute). Chi-squared tests were used to investigate differences in sociodemographic characteristics and medical comorbidities between the study and control groups. Additionally, Cox proportional hazard regression analysis was conducted to examine the relationship between ADT use and IDA risk during the 3-year study period. Data for patients who died or who were lost to follow-up during the study period were censored in the Cox regression. In total, 3152 patients died during the 3-year study period (2153 ADT users and 999 non-users). A two-sided $\mathrm{p}$ value $<0.05$ denoted statistical significance.

\section{Patient and public involvement}

Patients and the public were not involved in the development of the research question, outcome measures, study design and recruitment/conduct of the present study.

\section{RESULTS}

Data for patients' sociodemographic characteristics and medical comorbidities are shown in table 1 . No 
Table 1 Demographic characteristics of patients with prostate cancer stratified by the previous use or non-use of ADT $(n=10893)$

\begin{tabular}{|c|c|c|c|c|c|}
\hline \multirow[b]{2}{*}{ Characteristic } & \multicolumn{2}{|c|}{ Patients not receiving ADT $(n=3631)$} & \multicolumn{2}{|c|}{ Patients receiving ADT $(\mathrm{n}=7262)$} & \multirow[b]{2}{*}{$P$ value } \\
\hline & Total no & Column \% & Total no & Column \% & \\
\hline Age (years), mean (SD) & $74.26(8.81)$ & & $74.46(8.81)$ & & 0.284 \\
\hline Urbanisation level & & & & & 0.792 \\
\hline 1 (most urbanised) & 943 & 25.97 & 1816 & 25.01 & \\
\hline 2 & 871 & 23.99 & 1734 & 23.88 & \\
\hline 3 & 492 & 13.55 & 1024 & 14.10 & \\
\hline 4 & 492 & 13.55 & 1011 & 13.92 & \\
\hline 5 (least urbanised) & 833 & 22.94 & 1677 & 23.09 & \\
\hline Geographic region & & & & & 0.454 \\
\hline Northern & 1876 & 51.67 & 3676 & 50.62 & \\
\hline Central & 850 & 23.41 & 1688 & 23.24 & \\
\hline Southern & 860 & 23.68 & 1789 & 24.64 & \\
\hline Eastern & 45 & 1.24 & 109 & 1.50 & \\
\hline Monthly income & & & & & 0.385 \\
\hline NT\$0-15840 & 2426 & 66.81 & 4826 & 66.46 & \\
\hline NT\$15841-25000 & 932 & 25.67 & 1931 & 26.59 & \\
\hline$\geq N T \$ 25001$ & 273 & 7.52 & 505 & 6.95 & \\
\hline Hypertension & 2047 & 56.38 & 4099 & 56.44 & 0.946 \\
\hline Hyperlipidaemia & 718 & 19.77 & 1308 & 18.01 & 0.026 \\
\hline Diabetes & 792 & 21.81 & 1511 & 20.81 & 0.226 \\
\hline Coronary heart disease & 838 & 23.08 & 1679 & 23.12 & 0.962 \\
\hline Inflammatory bowel disease & 29 & 0.80 & 65 & 0.90 & 0.608 \\
\hline Gastrointestinal bleeding & 122 & 3.36 & 265 & 3.65 & 0.442 \\
\hline Previous cancers & 348 & 9.58 & 533 & 7.34 & $<0.001$ \\
\hline
\end{tabular}

The average exchange rate in 2015 was US $\$ 1.00 \approx N T \$ 30$.

ADT, androgen deprivation therapy; NT, New Taiwan.

statistically significant differences were found regarding age, monthly income, geographic location, residential urbanisation level and the incidence of hyperlipidaemia, diabetes, hypertension, coronary heart disease and inflammatory bowel disease between the study and control groups.

Table 2 Crude and adjusted HRs for anaemia among patients with prostate cancer during a 3-year follow-up period stratified by the previous use or non-use of ADT

\begin{tabular}{llll}
\hline Presence of iron-deficiency anaemia & $\begin{array}{l}\text { Total sample } \\
(\mathbf{n = 1 0 8 9 3 )}\end{array}$ & $\begin{array}{l}\text { Patients receiving ADT } \\
(\mathbf{n = 7 2 6 2})\end{array}$ & $\begin{array}{l}\text { Patients not receiving ADT } \\
(\mathbf{n}=3631)\end{array}$ \\
\hline Three-year follow-up period & & & $1.01(0.78-1.25)$ \\
\hline Incidence rate per 100 person-years $(95 \% \mathrm{Cl})$ & $1.45(1.29-1.61)$ & $1.66(1.45-1.86)$ & 1.00 \\
\hline Crude HR $(95 \% \mathrm{Cl})$ & -- & $1.64(1.25-2.14)$ & 1.00 \\
\hline Adjusted HR $(95 \% \mathrm{Cl})^{\star} \dagger$ & -- & $1.58(1.21-2.07)$ & 1.00 \\
\hline Adjusted HR $(95 \% \mathrm{Cl})^{*} \ddagger$ & -- & $1.62(1.24-2.12)$ & \\
\hline
\end{tabular}

*Using a Cox proportional regression with data censored if individuals died during the 3-year follow-up period.

†Adjustments were made for patients' geographic location, monthly income, urbanisation level, age and incidence of hyperlipidaemia, diabetes, hypertension, coronary heart disease and inflammatory bowel disease.

$\ddagger$ Adjustments were made for patients’ geographic location, monthly income, urbanisation level, age and incidence of hyperlipidaemia, diabetes, hypertension, coronary heart disease, inflammatory bowel disease, other cancers and gastrointestinal bleeding.

ADT, androgen deprivation therapy. 


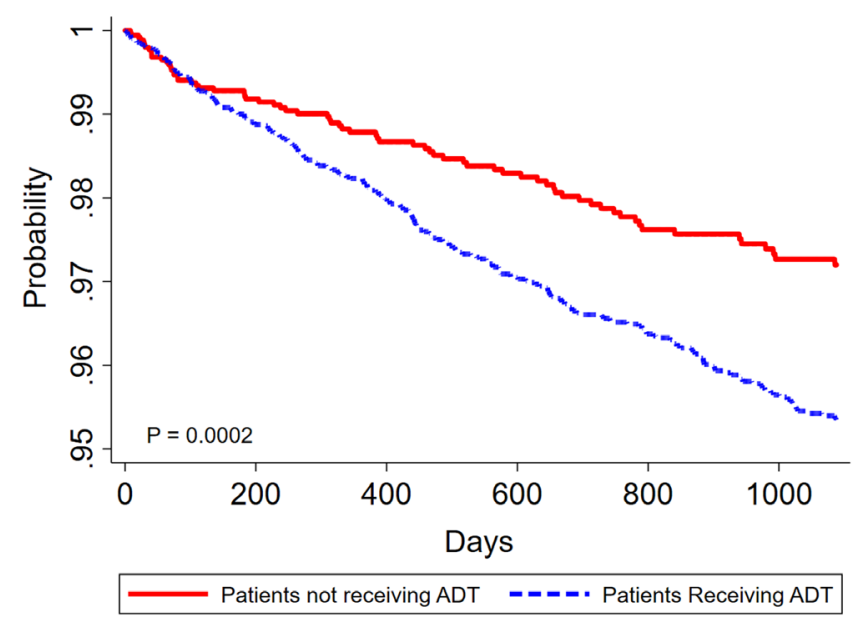

Figure 1 Kaplan-Meier curve of the risk of iron-deficiency anaemia IDA among patients with prostate cancer during a 3-year follow-up period as stratified by the previous use or non-use of ADT. ADT, androgen deprivation therapy; IDA, iron-deficiency anaemia.

The incidence of IDA during the 3 year study period is presented in table 2. The IDA incidence rate per 100 person-years for the entire 10,893-patient cohort was 1.45 (95\% CI 1.29 to 1.61 ). The incidence rates per 100 person-years for the study and control groups were 1.66 (95\% CI 1.45 to 1.86 ) and 1.01 (95\% CI 0.78 to 1.25 ), respectively. The log-rank test revealed that the study group had a significantly lower 3-year IDA-free survival rate than the control group ( $<<0.001$, figure 1$)$.

Table 2 further illustrates that the HR for IDA in the study group relative to the findings in the control group was 1.64 (95\% CI 1.25 to 2.14; $\mathrm{p} \leq 0.001)$. After adjusting for patients' age, monthly income, geographic location, residential urbanisation level and the incidence of hyperlipidaemia, diabetes, hypertension, coronary heart disease, inflammatory bowel disease, other cancers and gastrointestinal bleeding, the HR for the study group compared with the control group was 1.62 (95\% CI 1.24 to $2.12 ; \mathrm{p} \leq 0.001$ ). This study further classified patients in the study group as short-term and long-term ADT users based on the median duration of use. Compared with the findings in the control group, the adjusted HRs for shortterm ADT use in the study group were 2.06 (95\% CI 1.53 to 2.76) (table 3). Furthermore, sensitivity analyses were displayed in online supplementary tables 1 and 2.

\section{DISCUSSION}

To the best of our knowledge, this is the first study to investigate the relationship between ADT use in men with PCa and the 3-year IDA risk in an East Asian population. This study only included patients with PCa in the relevant analyses to avoid the potential effects attributable to cancer. To mitigate selection bias, a propensity score matching strategy (by year of the entry date, age, monthly income, geographical location, residential urbanisation level and medical comorbidities) was used to facilitate comparisons with patients with PCa who did not receive ADT. In this population-based retrospective cohort study of patients with PCa, a significant increase in IDA risk was observed with men treated with ADT.

The findings were consistent with those of prior Western studies. For instance, in the USA, Strum et al demonstrated that the haemoglobin levels of patients who received ADT for PCa declined from a mean of $149 \mathrm{~g} / \mathrm{L}$ at baseline to 139, 132 and $131 \mathrm{~g} / \mathrm{L}$ after 1, 2 and 3 months, respectively. ${ }^{30}$ In Canada, Timilshina et al performed an observational study of 250 patients with non-metastatic $\mathrm{PCa}$ and found that ADT was independently associated with a reduction of haemoglobin levels over 12 months. ${ }^{23}$ Among 110 patients with PCa who received ADT, one cohort study reported a decline in haemoglobin levels from $14.8 \mathrm{~g} / \mathrm{dL}$ at baseline to $12.9 \mathrm{~g} / \mathrm{dL}$ at evaluation. ${ }^{22}$ Another study of 72 patients with non-metastatic PCa who received adjuvant radiotherapy plus ADT reported

Table 3 Crude and adjusted HRs for anaemia among patients with prostate cancer during a 3-year follow-up period stratified by the duration of ADT

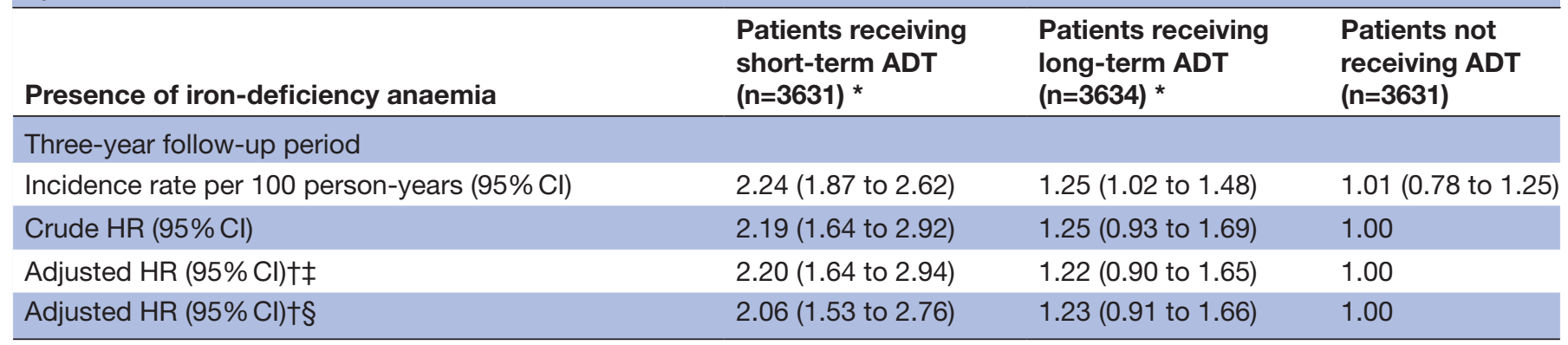

*We categorised the patients receiving ADT into two levels according to the median duration of ADT use (144 days).

†Using a Cox proportional regression with data censored if individuals died during the 3-year follow-up period;.

$\ddagger$ Adjustments were made for patients’ geographic location, monthly income, urbanisation level, age and incidence of hyperlipidaemia, diabetes, hypertension, coronary heart disease and inflammatory bowel disease.

$\S A$ djustments were made for patients' geographic location, monthly income, urbanisation level, age and incidence of hyperlipidaemia, diabetes, hypertension, coronary heart disease, inflammatory bowel disease, other cancers and gastrointestinal bleeding.

ADT, androgen deprivation therapy. 
that the haemoglobin level had significant declined after 2 years of androgen suppression. ${ }^{31}$ All of these studies investigated the association of ADT with haemoglobin levels, which is vital given that haemoglobin levels are important for identifying anaemia. However, these clinical studies included small sample sizes and featured a short duration. ${ }^{22} 233031$ Separately, Hicks et al performed a cohort study using the United Kingdom Clinical Practice Research Database linked to the Hospital Episode Statistics repository. Their findings revealed that patients with non-metastatic PCa who received ADT had a nearly threefold greater risk of anaemia than non-users $(\mathrm{HR}=2.90$, $95 \%$ CI 2.67 to 3.16$).{ }^{24}$ However, this study was performed in a western population, and generalisation of its findings to other ethnic groups is not possible.

Our study examined the association between ADT and the 3-year IDA risk among patients with PCa; however, the risk of anaemia caused by ADT use has remained unclear. To date, increasing biological evidence has suggested a potential connection between the receipt of ADT and subsequent IDA risk. ${ }^{32}$ A possible explanation for the underlying mechanism may be that androgen significantly increases the levels of bone marrow erythroid precursors and enhances the differentiation of bone marrow erythroid stem cells to erythrocytes. ${ }^{21} 33$ Furthermore, testosterone might influence erythropoietin secretion via peritubular fibroblast-like cells in the kidneys. ${ }^{34}$ In animal experiments, testosterone induced erythropoiesis-stimulating factor production in a bioassay using a mouse model of polycythaemia vera. ${ }^{35}$ Guo et al found that sensitivity to erythropoietin was induced and stress erythropoiesis was increased by testosterone, which upregulated the expression of GATA-binding protein 1 and GATA-dependent genes, in a study of ageing male mice. ${ }^{36}$ In addition, Bachman et al observed that treatment with testosterone in men was related to serum hepcidin suppression in a randomised double-blind clinical study. ${ }^{19}$ Testosterone directly interacts with the bone morphogenetic protein-Smad signalling pathway in hepatocytes and induces the downregulation of hepcidin transcription. ${ }^{37}$ Furthermore, these effects of androgen on hepatic hepcidin and renal erythropoietin gene expression could increase systemic iron transport and erythropoietin levels. ${ }^{37}$

The strengths of our study included the identification of the association between ADT use in patients with PCa and subsequent IDA risk using a large population-based database. The Taiwan NHIRD provides high accessibility to medical service data and adequate to detect IDA risk between the study and control groups. Moreover, the sociodemographic characteristics and medical comorbidities of patients with PCa were acknowledged as risk factors. Furthermore, we also used a propensity score matching strategy to eliminate potential biases such as patients' demographics and comorbidities between the study and control cohorts.

Several limitations of our retrospective study should be addressed. The study lacked information on potentially important patient characteristics, such as body mass index, smoking, nutrition and non-prescription medication use. Second, it is plausible that the database did not include all patients with PCa and IDA in Taiwan because some patients might have sought alternative medicines not recorded by the NHI programme and some patients with mild or normocytic anaemia may not have immediately sought medical treatment. Therefore, these patients might not have been captured via diagnosis codes. Third, information on the family history of anaemia, cancer stage and grade for the presence of metastases, chemotherapy, radiotherapy and surgery was unavailable this study. Finally, this study lacked information regarding blood variables. Nevertheless, this study identified patients with PCa who used ADT as cases and patients with PCa patients who did not use ADT as controls. In general, physicians in Taiwan perform complete blood counts for patients with PCa to identify suitable treatments. Consequently, we considered that these factors may not have affected the findings in this study.

\section{CONCLUSIONS}

In conclusion, the present study detected an increased IDA risk during a 3-year follow-up period among patients with $\mathrm{PCa}$ who received ADT. Medical professionals are recommended to be aware of the risk of anaemia following ADT. Clinicians and pharmacists need to consider the possible risk of IDA among patients with PCa who received $\mathrm{ADT}$ and assess the efficacy of preventative and treatment modalities for anaemia.

\section{Author affiliations}

${ }^{1}$ Department of Pharmacy, West Garden Hospital, Taipei, Taiwan

${ }^{2}$ Department of Pharmacy Practice, Tri-Service General Hospital, Taipei, Taiwan

${ }^{3}$ School of Pharmacy, National Defense Medical Center, Taipei, Taiwan

${ }^{4}$ Department of Pharmacology, National Defense Medical Center, Taipei, Taiwan

${ }^{5}$ Graduate Institute of Life Sciences, National Defense Medical Center, Taipei, Taiwan ${ }^{6}$ Department of Medical Research, Tri-Service General Hospital, National Defense

Medical Center, Taipei, Taiwan

${ }^{7}$ School of Public Health, National Defense Medical Center, Taipei, Taiwan ${ }^{8}$ Research Center of Biostatistics, Taipei Medical University, Taipei, Taiwan ${ }^{9}$ Emergency Department, China Medical University Hospital, Taichung City, Taiwan

${ }^{10}$ Department of Pharmacy, China Medical University Hospital, Taichung City, Taiwan

Contributors Conceptualisation: F-JW, Y-DC, I-HL and L-TK; Methodology: F-JW, Y-DC, Y-CL and L-TK; Formal analysis and investigation: F-JW and W-CC; Writing of original draft preparation: F-JW, J-HS, C-MC and Y-DC.

Funding The authors have not declared a specific grant for this research from any funding agency in the public, commercial or not-for-profit sectors.

Competing interests None declared.

Patient consent for publication Not required.

Ethics approval This study was exempt from full revision by the Tri-Service General Hospital Institutional Review Board (TSGHIRB No 2-105-05-082) because the dataset used was blinded to the public for research purposes.

Provenance and peer review Not commissioned; externally peer reviewed. Data availability statement No data are available. № additional data available. Open access This is an open access article distributed in accordance with the Creative Commons Attribution Non Commercial (CC BY-NC 4.0) license, which permits others to distribute, remix, adapt, build upon this work non-commercially, and license their derivative works on different terms, provided the original work is 
properly cited, appropriate credit is given, any changes made indicated, and the use is non-commercial. See: http://creativecommons.org/licenses/by-nc/4.0/.

\section{ORCID iD}

Li-Ting Kao http://orcid.org/0000-0003-0692-7408

\section{REFERENCES}

1 , Fitzmaurice C, Dicker D, et al, Global Burden of Disease Cancer Collaboration. The global burden of cancer 2013. JAMA Oncol 2015;1:505-27.

2 Attard G, Parker C, Eeles RA, et al. Prostate cancer. Lancet 2016;387:70-82.

3 Siegel RL, Miller KD, Jemal A. Cancer statistics, 2018. CA Cancer J Clin 2018;68:7-30.

4 Shahinian VB, Kuo Y-F, Gilbert SM. Reimbursement policy and androgen-deprivation therapy for prostate cancer. N Engl J Med 2010;363:1822-32

5 Bryant AK, D'Amico AV, Nguyen PL, et al. Three-Month posttreatment prostate-specific antigen level as a biomarker of treatment response in patients with intermediate-risk or high-risk prostate cancer treated with androgen deprivation therapy and radiotherapy. Cancer 2018;124:2939-47.

6 Harris WP, Mostaghel EA, Nelson PS, et al. Androgen deprivation therapy: progress in understanding mechanisms of resistance and optimizing androgen depletion. Nat Clin Pract Urol 2009;6:76-85.

7 Owen PJ, Daly RM, Livingston PM, et al. Lifestyle guidelines for managing adverse effects on bone health and body composition in men treated with androgen deprivation therapy for prostate cancer: an update. Prostate Cancer Prostatic Dis 2017;20:137-45.

8 Nguyen PL, Alibhai SMH, Basaria S, et al. Adverse effects of androgen deprivation therapy and strategies to mitigate them. Eur Urol 2015:67:825-36.

9 Basaria S, Muller DC, Carducci MA, et al. Hyperglycemia and insulin resistance in men with prostate carcinoma who receive androgendeprivation therapy. Cancer 2006;106:581-8.

10 Grossmann M, Hamilton EJ, Gilfillan C, et al. Bone and metabolic health in patients with non-metastatic prostate cancer who are receiving androgen deprivation therapy. Med J Aust 2011;194:301-6.

11 Sharifi N, Gulley JL, Dahut WL. Androgen deprivation therapy for prostate cancer. JAMA 2005;294:238-44.

12 Wu F-J, Sheu S-Y, Lin H-C, et al. Increased fall risk in patients receiving androgen deprivation therapy for prostate cancer. Urology 2016;95:145-50.

13 Johnson-Wimbley TD, Graham DY. Diagnosis and management of iron deficiency anemia in the 21st century. Therap Adv Gastroenterol 2011;4:177-84

14 Caro JJ, Salas M, Ward A, et al. Anemia as an independent prognostic factor for survival in patients with cancer: a systemic, quantitative review. Cancer 2001;91:2214-21.

15 Dicato M, Plawny L, Diederich M. Anemia in cancer. Ann Oncol 2010;21 Suppl 7:vii167-72.

16 Dueregger A, Heidegger I, Ofer P, et al. The use of dietary supplements to alleviate androgen deprivation therapy side effects during prostate cancer treatment. Nutrients 2014;6:4491-519.

17 Lopez A, Cacoub P, Macdougall IC, et al. Iron deficiency anaemia. Lancet 2016;387:907-16.

18 Ferrucci L, Maggio M, Bandinelli S, et al. Low testosterone levels and the risk of anemia in older men and women. Arch Intern Med 2006;166:1380-8.
19 Bachman E, Feng R, Travison T, et al. Testosterone suppresses hepcidin in men: a potential mechanism for testosterone-induced erythrocytosis. J Clin Endocrinol Metab 2010;95:4743-7.

20 Latour C, Kautz L, Besson-Fournier C, et al. Testosterone perturbs systemic iron balance through activation of epidermal growth factor receptor signaling in the liver and repression of hepcidin. Hepatology 2014;59:683-94.

21 Moriyama Y, Fisher JW. Effects of testosterone and erythropoietin on erythroid colony formation in human bone marrow cultures. Blood 1975;45:665-70.

22 D'Amico AV, Saegaert T, Chen M-H, et al. Initial decline in hemoglobin during neoadjuvant hormonal therapy predicts for early prostate specific antigen failure following radiation and hormonal therapy for patients with intermediate and high-risk prostate cancer. Cancer 2002;95:275-80.

23 Timilshina N, Hussain S, Breunis $\mathrm{H}$, et al. Predictors of hemoglobin decline in non-metastatic prostate cancer patients on androgen deprivation therapy: a matched cohort study. Support Care Cancer 2011;19:1815-21.

24 Hicks BM, Klil-Drori AJ, Yin H, et al. Androgen deprivation therapy and the risk of anemia in men with prostate cancer. Epidemiology 2017;28:712-8.

25 Rebbeck TR. Prostate cancer genetics: variation by race, ethnicity, and geography. Semin Radiat Oncol 2017;27:3-10.

26 Taitt HE, Trends G. Global trends and prostate cancer: a review of incidence, detection, and mortality as influenced by race, ethnicity, and geographic location. Am J Mens Health 2018;12:1807-23.

27 McLean E, Cogswell M, Egli I, et al. Worldwide prevalence of anaemia, who vitamin and mineral nutrition information system, 1993-2005. Public Health Nutr 2009;12:444-54.

28 Poon DMC, Chan T, Chan K, et al. Preliminary efficacy and tolerability of chemohormonal therapy in metastatic hormone-naïve prostate cancer: the first real-life experience in Asia. Asia Pac J Clin Oncol 2018;14:347-52.

29 Liu CY, Hung YT, Chuang YL, et al. Incorporating development stratification of Taiwan townships into sampling design of large scale health interview survey. J Health Manag 2006;4:1-22.

30 Strum SB, McDermed JE, Scholz MC, et al. Anaemia associated with androgen deprivation in patients with prostate cancer receiving combined hormone blockade. Br J Urol 1997;79:933-41.

31 Choo R, Chander S, Danjoux C, et al. How are hemoglobin levels affected by androgen deprivation in non-metastatic prostate cancer patients? Can J Urol 2005;12:2547-52.

32 Shahani S, Braga-Basaria M, Maggio M, et al. Androgens and erythropoiesis: past and present. J Endocrinol Invest 2009;32:704-16.

33 Dai D, Han S, Li L, et al. Anemia is associated with poor outcomes of metastatic castration-resistant prostate cancer, a systematic review and meta-analysis. Am J Transl Res 2018;10:3877-86.

34 Pan X, Suzuki N, Hirano I, et al. Isolation and characterization of renal erythropoietin-producing cells from genetically produced anemia mice. PLoS One 2011;6:e25839.

35 Mirand EA, Gordon AS, Wenig J. Mechanism of testosterone action in erythropoiesis. Nature 1965;206:270-2.

36 Guo W, Li M, Bhasin S. Testosterone supplementation improves anemia in aging male mice. J Gerontol A Biol Sci Med Sci 2014;69:505-13.

37 Guo W, Bachman E, Li M, et al. Testosterone administration inhibits hepcidin transcription and is associated with increased iron incorporation into red blood cells. Aging Cell 2013;12:280-91. 